\title{
The Application and Training of Mask Resonance in Singing
}

\author{
Shanshan Jiang \\ School of Music \\ Heihe University \\ Heihe, China 164300
}

\begin{abstract}
Mask resonance is known as the essence and the key of bel canto. Get a good mask resonance, sound with high harmonics, penetrating power, vibration, endurance and other characteristics. In order to obtain a good mask resonance, it needs to have good breathing and diaphragm support state, throat pharyngeal state and humming state.
\end{abstract}

Keywords -singing; mask resonance; mask singing

\section{INTRODUCTION}

Singing is a skill to solve the voice beauty of the singer, is the artistic crystallization of singers' and educators' singing practice through the thousands of years, not out of thin air. As which singing is better and which singing is not good, that is depend on the training methods and the quality of the trainer's voice, but also depend on the style of the sound. As to like the back voice, no one will force you to change, because music is like this, it is up to your hearing and your imagination. As for the individual authors and experts believe that mask singing, nasal resonance, head cavity resonance is unscientific utterance theory.

\section{MEANING OF MASK SINGING METHOD}

Mask singing method is a scientific singing method in Bel canto system. The nomenclature of the mask was derived from the mask that French nobles often wear in the court balls during the 16th century. The mask singing advocated by Resque at the time is a vocal method mainly giving full play of nasal resonance. He said: "The sound should not in the nose, but the nose should in the sound." Its main purpose is to get the mask resonance, mask resonance areas are above the cheeks, from both sides of the temple to the eyebrows, forehead, frontal sinuses and sinus bone on both sides of the nose all have resonance. For the mask singing training, the one must master the basic requirements of each process, lay a good basic skill, so that to master the mask singing.

\section{MASK SINGING TRAINING}

Mask singing as the traditional Italian singing method, it has its own training system, requirements and training means. The training of breath, resonance, acoustic area transition and head tone must follow the method of mask singing training so that it could get the vocal sound, resonance and quality with

Fund Project: 2016 Heilongjiang province philosophy and social science research planning project "Scientific training of voice and health research"; Project number: 16YSC03. the advantages of mask singing, and achieve the training purpose. Mask training, with particular emphasis on the breath maintain and mixed sound method, for the breath maintain most of the trainers despite have different breathing methods, but the mask resonance stressed abdominal breathing. For acoustic area transition, it must use mixed sound method in order to obtain the natural transition from sound zone to soprano zone, the mixed sound method is the key to mask singing. First of all, it must be very careful, muse under the guidance of experienced teachers. Secondly, it could only been obtained under the support of good breathing. If not get mixed sound, the sound does not mix up, everything will be out of the question. Therefore, the training of mixed sound is the most important process of mask singing. In addition, in the mixed sound training phase if can not successfully complete, do not look for other reasons, find the reason from the breath maintenance. If breath is not in good control, mixed sound skill is impossible to master. Therefore, for the mask singing training, it requires the mastery of each training process, lay a good basic skill, so that to master the mask singing.

\section{ABDominal BREATHING TRAINING OF MASK SingING}

Respiratory support is a very important skill in vocal skill training. Regardless of the methods used for sound training, breathing is always the foundation. In Italy, there is a proverb about the importance of breathing in singing: who controlled breathing, and who learned how to sing. In China's ancient vocal music skills there are also the classic theory of: "Those who are good at singing must arrange their breath first." It can be seen that both Chinese and foreign all have descriptions of the importance of breathing for vocal skills and they all paid adequate attention to that.

For beginners, abdominal breathing training requirements are, first upright the body, hands crossed on the back of the neck, two feet apart, inhale, when inhale can use both mouth and nose, and then slowly squat body, pay attention to back straight, always maintain a sense of expansion around the waist, upper teeth align lower teeth, exhale the gas and make si si sound from the upper and lower incisors gap, pay attention the exhale should be even until exhale all the gas and fully squat down, and then quickly straightened up, inhale with nose, attention it should be fast. Make round-trip repetitive training. 20 minutes a day or so, roughly half a month before the breathing exercises of sound training start. Breathing 
exercises for vocal training. It must pay attention to three issues, one is to find the focus spot of the mask; second is lower the breath, placed it in the lower abdomen pubic region; third is the chest must to suck open. Use sound training songs to make voice training. Breath should be controlled by pubic gas and make the breathing movement very even, after familiar can practice with wave-type breathing movement method and gradually experience the downward movement in pubic region and the voice upward concentration feeling, the tension between them is approximate to the elastic rubber-like movement, known as Laqi. Usually should insist on playing $\mathrm{DuRu}$ practice, not overnight, as long as you like singing, you must insist on playing DuLu practice. This breathing practice method has three benefits, first it practiced the breath control ability, second it could make massage for the vocal cords, third is you can feel the pressure of the abdomen. Just like physical activity before the match. Slowly you will gradually realize that one you practice $\mathrm{Du} \mathrm{Lu}$, you abdominal pubic region will naturally tension, rather than take the initiative to do, it is a passive natural breathing exercise. For such breathing exercises, you must guard against the breath holding, uneven exhale, tight chest, couldn't find the resonance focus. After find the focal point of the mask resonance, along the back of the nose bridge under the support of breath and movement make the sound forward upside down so that to make preparation for the later mixed sound and transition. In addition, after having the basic stability of such breathing exercises, you should gradually remind the learners to realize the separation of sound and breath. The sound at the mask focus point should be disconnected with the breath from lower chest and low breath support. It should make tangible chest becomes invisible, formed the high positioned sound under breath support. Everything is come from ideas and imagination, reaching the point of ecstasy. All of these breathing exercises are built a solid foundation for later technology and mixed sound, we must not be ambitious, each step must be very careful and cautions, serious treat every step and process of breathing, take every step good and solid in order to carry out the next step of training, the middle low voice zone training.

\section{The Middle Low Voice Zone Training}

Mask singing method also has very strict requirements for the middle low voice zone training, if can not lay a good foundation with middle low voice zone, then mask resonance simply impossible to get, and even all the trainings before may go down the drain, or even couldn't complete mixed sound and acoustic area transition steps, so that to make mask singing more than just talk. The middle low voice zone normally for tenor or soprano refers to the sound treble in the range of a to $\mathrm{c} 1$, the sound of this range often has a bigger proportion of natural voice for the sounder, also is the sound which the general singing voice easy to sing. But giving a sound with certain quality is not an easy thing. For the importance of middle voice training, most vocal music educationists and singers have their unique insights. To enhance the physiological function of the learner's voice, only after pull out the learner's gene resonance, then it is possible to conduct mixed sound training, and finally reach to harmonic resonance. Vocal skill training in fact from the resonance point of view is to train students how to from pitch resonance to harmonic resonance, and obtain a high positioned mixed head tone. Whichever method you use, obtaining high-frequency harmonics is the end result of vocal skill training. However, if the pitch resonance training is not good, it is impossible to obtain the head tone of high harmonic resonance. So the middle lower voice zone training of mask singing method in fact is training students' pitch resonance. At this point, the teacher's auditory experience is the sound is more open, and even some white, but this is a necessary step, do not rush, we must practice the pitch well. The general impatient learners may think why Mask singing trainings are all natural sound, why it does not like the general soprano or tenor? In particular the soprano, often think why there is no falsetto? And even some 1 experienced teachers will have to ask such questions, does the sound too white? Also are all natural sound, how to sing? It is better to think of other ways, first find out the falsetto, or else continue to sing in this way is incredibly. In fact, mask singing is in such training steps, sound comes up little by little, adds the falsetto step by step. Gradually make harmonic proportion between the natural sound and falsetto, can not transit too quickly and suddenly, if so, often in the auditory it will make people feel the natural and falsetto convergence is not natural.

\section{ACOUSTIC AREA TRANSITION AND MiXED SOUND TRAINING}

Before process the mixed sound training of mask singing method, you must first find out the "point" problem in mask singing. For the "point" various works have discussed it, Lan Pilty called it as the focus of sound, he believes this sound focus is in the middle of the skull. The believers of closed singing believe it is in the back of the head and so on. In the case the "point" appears as a noun because of the concentration of sound. Although the term has different understandings and practical experiences, we just put aside for the time being. First look at the physical property of point, "point" concept is relative emerged from the concept of "surface". The space range of "surface" is larger than the space range of "point", so from the perspective of sound, "point" refers to the gathering of sound, "surface" refers to the sound diffusion. From the perspective of the breathing density, the air density of "point" is much larger than the air density of "surface". In terms of sound resonance, the frequency of sound vibration is determined by the air density and space. The smaller is the space, the denser is the air, the faster is the frequency of sound vibration, and correspondingly the more is the harmonics resonance. So in terms of vocal, this "point" bears the task of harmonic "point". In addition, the location of sound, as for the history of vocal development experience, the higher the position, the easier the sound into the head tone, the easier the singing is, the more beautiful the sound is. This has become a recognized truth in the vocal music industry, open all kinds of works you can find it everywhere, this article will not repeat them here. But the "point" position mentioned in mask singing method is the frontal sinus, located between the two eyebrows, also known as the focal point of the mask resonance. The purpose of acoustic area transition is to convert the sound transition to head tone. It is to combine the natural sound and falsetto together and make appropriate adjustment for the ratio, then enter the ideal state of the head tone without leaving any 
trace. Thus, the "point" here also bears the task to transit from middle low voice zone to high-pitched voice zone. Therefore, this "point" also has the ratio adjustment task between natural sound and falsetto. It is because this task, so Mask singing method called this focal "point" as the mixed sound point. Thus, the importance of the "point" is self-evident, also the problem of the natural sound and falsetto in voice is an important problem that can not be avoid in mixed sound.

\section{CONCLUSION}

Mask singing training is voice training, and training for the singer to master the breath, middle low voice zone, acoustic area transition and mixed sound skills. Completion of mixed sound skills training is not equal to the end of mask singing training. In order to make proficiency to use the mask singing method, you must also perform "fine tuning" during the exercise. Fine-tuning is the adjustment, is trained the ability to adjust the voice. For example: before the issue of each voice, you must think it through, what kind of voice is required by bel canto, even after the voice is issued, immediately feel wrong, it is necessary to promptly adjust the focus on the breath or voice, so that the subsequent voice issued will meet the requirements of bel canto. It means the voice must be controlled by the singers, so that they could give out and end freely. Take a metaphor it is like remote sensing satellite, not only we have to launch the satellite, but also we should adjust its orbit and posture, and even not only launch satellite, but also recover it after it complete its mission.

We should remote control everything about the satellite, we can not leave it alone once launched it. The same is true with voice, and the highest skill is the control of voice. Strength to give a little more, a little less, resonance a little more or a little less and so on all can be controlled and adjusted. In addition, for the practice, breath should go before the voice, during skip sound exercises, the movement of lower abdomen should be elastic movement like dog breathing, teeth should open, make a smile, etc., are all effective language in the practice of mask singing method, the purpose is to inspire students to learn faster and better to master the singing method. It can be seen that the mask singing training is a training to make the singer's voice more beautiful, to unified voice zone, to master the voice intensity, volume and tone. It is a scientific bel Canto singing method, not out of thin air. The voice training is an overall, systematic, unique skills training. It is a scientific bel Canto singing method which proved by singers' and educators' singing practice through the hundreds of years in many countries. It is the essence of the Italian vocal singing and long-standing singing tradition.

\section{REFERENCES}

[1] Liu Min. Effective Ways to Obtain Mask Resonance in Singing [J]. Journal of Guizhou University (Art Edition), 2010 (1): 40-43.

[2] Wang Hongwei. Discussion on Mask resonance in Bel Canto singing[J]. Popular Literature, 2013 (12): 244-244. 